\title{
Robust Control Allocation for Spacecraft Attitude Stabilization under Actuator Faults and Uncertainty
}

\author{
Aihua Zhang, ${ }^{1}$ Yongchao Wang, ${ }^{1}$ Zhiqiang Zhang, ${ }^{1}$ and Hamid Reza Karimi ${ }^{2}$ \\ ${ }^{1}$ College of Engineering, Bohai University, Jinzhou 121013, China \\ ${ }^{2}$ Department of Engineering, Faculty of Engineering and Science, the University of Agder, 4898 Grimstad, Norway \\ Correspondence should be addressed to Aihua Zhang; jsxinxi_zah@163.com
}

Received 21 November 2013; Accepted 11 December 2013; Published 13 February 2014

Academic Editor: Weichao Sun

Copyright (c) 2014 Aihua Zhang et al. This is an open access article distributed under the Creative Commons Attribution License, which permits unrestricted use, distribution, and reproduction in any medium, provided the original work is properly cited.

\begin{abstract}
A robust control allocation scheme is developed for rigid spacecraft attitude stabilization in the presence of actuator partial loss fault, actuator failure, and actuator misalignment. First, a neural network fault detection scheme is proposed, Second, an adaptive attitude tracking strategy is employed which can realize fault tolerance control under the actuator partial loss and actuator failure within $\lambda_{\text {min }}=0.5$. The attitude tracking and faults detection are always here during the procedure. Once the fault occurred which could not guaranteed the attitude stable for $30 \mathrm{~s}$, the robust control allocation strategy is generated automatically. The robust control allocation compensates the control effectiveness uncertainty which caused the actuator misalignment. The unknown disturbances, uncertain inertia matrix, and even actuator error with limited actuators are all considered in the controller design process. All are achieved with inexpensive online computations. Numerical results are also presented that not only highlight the closed-loop performance benefits of the control law derived here but also illustrate its great robustness.
\end{abstract}

\section{Introduction}

The stabilization of spacecraft attitude is one of the fundamental manoeuvres and primary attitude control tasks that any spacecraft needs to frequently perform during its mission. Such a response should be achieved globally in the presence of external disturbances, modelling uncertainties, subsystem failures, and limited resources. It is really a challenging undertaking especially when all those issues are treated simultaneously. And hence fault tolerant control (FTC) for rigid spacecraft attitude control has been carried out during the past decades. FTC is a control design strategy that guarantees system stability and acceptable performance [1]. The passive FTC [2-4] and active FTC [5] are the two typical approaches of the FTC. The passive FTC has a very limited faults tolerance capability for its conservative design [6-8]. On the contrast, the active FTC compensates the drawback via synthesizing or selecting the new controller online [9]. In general, the active FTC should confirm the faults, in real time, which requires a fault detection and diagnosis (FDD) mechanism. The errors in fault detection and time delays between the fault detection and controller reconfiguration all may generate the system to be uncontrolled [10].

Recently, a great deal of attention on fault tolerant controller designs have been paid for spacecraft attitude control $[11,12]$. Boyarko et al. [13] employed dynamics inversion and time-delay theory to design attitude tracking control for a rigid satellite actuated by four momentum wheels. The shortcoming of $[11,12]$ is the fact that the full knowledge of the actuator faults was assumed to be known exactly under the external disturbances free. Cai et al. [14] updated bounds on lumped parameter perturbations but only considered failures that are modelled as the loss of effectiveness of thrusters. A control augmentation method, similar to adaptive FTC [1518], for the attitude tracking control of flexible spacecraft is proposed in [19]. Persistent bounded disturbances and unknown inertia parameter uncertainties were explicitly considered using an adaptive backstepping control. Although their approaches also compensated for the additive and partial loss of effectiveness faults of reaction wheel using sliding mode control, the actuator uncertainty is ignored. Active FTC has also been used for spacecraft, as suggested 
in [20-22] and the references therein. Hou et al. [23] applied a two-stage Kalman filtering algorithm to estimate reaction flywheel and sensor faults and then designed two faulttolerant controller to compensate for the constant loss of actuator effectiveness on the nominal system. However, these references can only treat a single type of spacecraft actuator fault, and there are few methods able to handle additive faults and the loss of actuator effectiveness simultaneously in present of the external disturbances. Optimal use of the large amount of actuators will be a solution.

Control allocation is one approach to managing the actuator redundancy for different control strategies handling actuator faults [24, 25]. Dynamic control allocation [26], which is used in the real systems, is to distribute the control command in dynamic sense. The control demand determined in the form of finite difference equations over a certain time interval. Current control allocation methods include direct allocation, optimization-based allocation, and some simple allocation methods. But all of these methods can do nothing in presence of the time-varying partial loss faults and actuator errors. Thus, amount of attention on this issue above has been discussed in recent years.

Therefore, from that point of view, in this study, a novel fault tolerant robust optimal control strategy for allocation is developed for the problem of rigid spacecraft attitude stabilization under actuator faults and uncertainties. Also, the potential effects of external disturbances and uncertain moment inertia on system performance are explicitly considered during the whole controller design process. In contrast to the most existing fault tolerant attitude controller, the merit of the proposed control law is the fact that we can control the robust control allocation. We can judge the states of the actuator partial loss fault via detection of the tracking performance index $I_{e}$. If the three fly reaction flywheels are able to produce a combined force sufficient enough to allow the spacecraft to perform the given manoeuvres, the robust control allocation will not be generated. The adaptive attitude tracking control can ensure the stable attitude tracking with guaranteed performance. Otherwise, the robust control allocation is generated immediately if the fault has been for $30 \mathrm{~s}$. During the whole control procedure, the attitude tracking and fault detection with the simple design and inexpensive online computations, a feature of practical importance for real-time implementation especially when onboard memory space and computing power, are limited. And the robust control allocation can settle the problem of control effectiveness uncertainty which caused the timevarying partial loss fault. The benefits of the proposed control methods are analytically authenticated and also validated via numerical simulation study.

The remainder of this paper is organized as follows. Section 2 summarizes the mathematical model of rigid spacecraft attitude and its control problems. Section 3 presents the proposed neural network fault detection method and the attitude tracking control scheme in the presence of uncertain inertia parameters, external disturbances, and partial loss fault, actuator failures. The robust optimal control allocation strategy for the uncertainty of the control effectiveness is discussed in Section 3.2. Simulation results to demonstrate various features of the proposed scheme are given in Section 4 followed by conclusion in Section 5 .

\section{Mathematical Model of a Spacecraft and the Control Problem}

2.1. Kinematics and Dynamics Equation. In this paper, the nonlinear equations of attitude motion along the body fixed control axes, in term of attitude kinematics and the spacecraft dynamics, are given by

$$
\begin{gathered}
J \dot{\omega}+\omega^{\times} J \omega=u+d \\
Q=\left[\begin{array}{c}
q_{0} \\
q^{T}
\end{array}\right]=\left[\begin{array}{c}
\cos \left(\frac{\Phi}{2}\right) \\
n \sin \left(\frac{\Phi}{2}\right)
\end{array}\right],
\end{gathered}
$$

with $n$ and $\Phi$ denoting the Euler axis and the Euler angle, respectively, and the following constraint is satisfied:

$$
q^{T} q+q_{0}^{2}=1
$$

Then, the kinematical differential equation in terms of unit quaternion is given by [27]

$$
\begin{gathered}
\dot{q}_{0}=-\frac{1}{2} q^{T} \omega \\
\dot{q}=\frac{1}{2}\left(q_{0} I_{3}+q^{\times}\right) \omega,
\end{gathered}
$$

where $\omega \in \mathbb{R}^{3}$ is the angular velocity of the spacecraft bodyfixed frame to the inertial frame $\mathscr{I}$ and expressed in the bodyfixed frame $\mathscr{B}$ and $u \in \mathbb{R}^{3}$ and $d \in \mathbb{R}^{3}$ demote the control torques and external disturbances, respectively. $J \in \mathbb{R}^{3}$ is the inertia matrix of the spacecraft expressed in $\mathscr{B} . I_{3}$ is the $3 \times$ 3 identity matrix, and $\omega^{\times}$denotes a skew-symmetric matrix which is given by

$$
\omega^{\times}=\left[\begin{array}{ccc}
0 & -\omega_{3} & \omega_{2} \\
\omega_{3} & 0 & -\omega_{1} \\
-\omega_{2} & \omega_{1} & 0
\end{array}\right] .
$$

2.2. Spacecraft Attitude Tracking Model. During the mission of spacecraft attitude tracking, the desired attitude of the spacecraft is described with a desired frame $\mathscr{D}$ with respect to the inertial frame $\mathscr{I}$ being specified by the desired unit quaternion $Q_{d}=\left(q_{d 0}, q_{d}^{T}\right) \in \mathbb{R} \times \mathbb{R}^{3}$. The angular velocity of $\mathscr{D}$ with respect to $\mathscr{I}$ expressed in $\mathscr{D}$ is denoted by $\omega_{d} \in \mathbb{R}^{3}$. Then, we define the attitude tracking error quaternion $Q_{e}=$ $\left(e_{0}, e^{T}\right) \in \mathbb{R} \times \mathbb{R}^{3}$ as the relative orientation between the body frame $\mathscr{B}$ and the desired frame $\mathscr{D}$, which is given by

$$
\begin{gathered}
e=q_{d 0} q-q_{0} q_{d}+q^{\times} q_{d} \\
e_{0}=q_{d 0} q+q_{d}^{T} q
\end{gathered}
$$

and the following constraints are satisfied:

$$
\begin{gathered}
q_{d}^{T} q_{d}+q_{d 0}^{2}=1 \\
e^{T} e+e_{0}^{2}=1
\end{gathered}
$$


$\omega_{e} \in \mathbb{R}^{3}$ represents the corresponding error angular velocity; then it has

$$
\omega_{e}=\omega-R \omega_{d}
$$

where $R \in \mathbb{R}^{3 \times 3}$ denotes the corresponding rotation matrix that brings $\mathscr{D}$ onto $\mathscr{B}$, and

$$
R=\left(e_{0}^{2}-e^{T} e\right) I_{3}+2 e e^{T}-2 e_{0} e^{\times}
$$

with (1)-(9), tracking error dynamic equation can be obtained as follows:

$$
\begin{gathered}
J \dot{\omega}_{e}=-\omega^{\times} J \omega+u+d+J\left(\omega_{e}^{\times} R \omega_{d}-R \dot{\omega}_{d}\right) \\
\dot{e}_{0}=-\frac{1}{2} e^{T} \omega_{e} \\
\dot{e}=\frac{1}{2}\left(e^{\times}+e_{0} I_{3}\right) \omega_{e} .
\end{gathered}
$$

2.3. Spacecraft Dynamic with Actuator Fault. For spacecraft, the control torque command for three body-fixed reference frames is generated by a combination of multiple wheels, usually four-wheel sets. Let us consider reaction flywheel control which is with typical four inclined outfit. Three reaction wheels rotation axes are orthogonal to the spacecraft ontology shaft and the fourth flywheel is installed with the equiangular direction with the ontology three axis.

When we employ the configuration of four reaction wheels shown in Figure 1, the spacecraft dynamics equation (1) should be transformed into this following form:

$$
J \dot{\omega}+\omega^{\times} J \omega=L^{*} \tau_{i}+d
$$

where $\tau=\left[\begin{array}{llll}\tau_{1} & \tau_{2} & \tau_{3} & \underbrace{\tau_{4}}_{\text {redundancy }}\end{array}\right]^{T}$ and $L^{*}$ denotes the control effectiveness matrix.

At present, control efficiency matrix $L^{*}$ is supposed to be accurate and correct, but it is uncertain facing the reality such as actuator error. The source of the actuator error comes from two parts: one is misalignment for which is assumed that the reaction flywheel is tilted over nominal reaction flywheel direction with small constant angles, $\Delta \alpha$ and $\Delta \beta$ as shown in Figure 2. The other is magnitude error which is a small random variable in practical problem. The real reaction flywheel force with magnitude error and misalignment is expressed as the sum of the nominal and reaction flywheel terms in the body frame

$$
\begin{aligned}
u= & \left(\tau_{1}+\Delta \tau_{1}\right)\left[\begin{array}{c}
\cos \Delta \alpha_{1} \\
\sin \Delta \alpha_{1} \cos \Delta \beta_{1} \\
\sin \Delta \alpha_{1} \sin \Delta \beta_{1}
\end{array}\right] \\
& +\left(\tau_{2}+\Delta \tau_{2}\right)\left[\begin{array}{c}
\sin \Delta \alpha_{2} \cos \Delta \beta_{2} \\
\cos \Delta \alpha_{2} \\
\sin \Delta \alpha_{2} \sin \Delta \beta_{2}
\end{array}\right]
\end{aligned}
$$

$$
\begin{aligned}
& +\left(\tau_{3}+\Delta \tau_{3}\right)\left[\begin{array}{c}
\sin \Delta \alpha_{3} \cos \Delta \beta_{3} \\
\sin \Delta \alpha_{3} \sin \Delta \beta_{3} \\
\cos \Delta \alpha_{3}
\end{array}\right] \\
& +\underbrace{\left(\tau_{4}+\Delta \tau_{4}\right)\left[\begin{array}{c}
\cos \left(\alpha_{4}+\Delta \alpha_{4}\right) \cos \left(\beta_{4}+\Delta \beta_{4}\right) \\
\cos \left(\alpha_{4}+\Delta \alpha_{4}\right) \sin \left(\beta_{4}+\Delta \beta_{4}\right) \\
\sin \left(\alpha_{4}+\Delta \alpha_{4}\right)
\end{array}\right]}_{\text {redundancy }} .
\end{aligned}
$$

In addition, the following relationships are adopted to approximate (14):

$$
\cos \Delta \alpha \approx \cos \Delta \beta \approx 1, \quad \sin \Delta \alpha \approx \Delta \alpha, \quad \sin \Delta \beta \approx \Delta \beta .
$$

We transform (14) into the following form:

$$
u=L_{0}(\tau+\Delta \tau)+\Delta L(\tau+\Delta \tau)
$$

where $\Delta L, \tau+\Delta \tau$ is the attitude control torque defined by

$$
\begin{aligned}
& \Delta L=\left[\begin{array}{ll}
\Delta L_{1} & \underbrace{\Delta L_{2}}_{\text {redundancy }}
\end{array}\right] \\
& \tau+\Delta \tau=\left[\begin{array}{llll}
\tau_{1}+\Delta \tau_{1} & \tau_{2}+\Delta \tau_{2} & \tau_{3}+\Delta \tau_{3} & \underbrace{\tau_{4}+\Delta \tau_{4}}_{\text {redundancy }}
\end{array}\right]^{T}, \\
& L_{0}=\left[\begin{array}{cccc}
1 & 0 & 0 & \cos \alpha_{4} \cos \beta_{4} \\
0 & 1 & 0 & \cos \alpha_{4} \sin \beta_{4} \\
0 & 0 & 1 & \underbrace{\sin \alpha_{4}}_{\text {redundancy }}
\end{array}\right] \\
& \Delta L_{1}=\left[\begin{array}{ccc}
0 & \Delta \alpha_{2} \cos \Delta \beta_{2} & \Delta \alpha_{3} \cos \Delta \beta_{3} \\
\Delta \alpha_{1} \cos \Delta \beta_{1} & 0 & \Delta \alpha_{3} \sin \Delta \beta_{3} \\
\Delta \alpha_{1} \sin \Delta \beta_{1} & \Delta \alpha_{2} \sin \Delta \beta_{2} & 0
\end{array}\right] \\
& \Delta L_{2}=\left[\begin{array}{c}
-\Delta \alpha_{4} \sin \alpha_{4} \cos \beta_{4}-\Delta \beta_{4} \cos \alpha_{4} \sin \beta_{4} \\
-\Delta \alpha_{4} \sin \alpha_{4} \sin \beta_{4}+\Delta \beta_{4} \cos \alpha_{4} \cos \beta_{4} \\
\Delta \alpha_{4} \cos \alpha_{4}
\end{array}\right] .
\end{aligned}
$$

In order to simulate the magnitude error enough, we assume the magnitude error as Gaussian random process to descript the relation between the output torque and magnitude error, and the magnitude error can be written as

$$
\Delta \tau= \begin{cases}0.1|\tau| r(t) & |\tau|>0.03 \\ 0.03 r(t) & |\tau| \leq 0.03\end{cases}
$$

We can get this piece of information from (22) that the magnitude error would be employed $10 \%$ random error if the control torque is bigger in some direction; otherwise, it has the constant magnitude random error. This case is in accordance with the practice.

By some algebraic manipulation with (16), (13) can be rewritten as

$$
\begin{gathered}
J \dot{\omega}+\omega^{\times} J \omega=L_{0}(\tau+\Delta \tau)+\Delta L(\tau+\Delta \tau)+d \\
J \dot{\omega}+\omega^{\times} J \omega=\underbrace{\left(L_{0}+\Delta L\right)}_{L}(\tau+\Delta \tau)+d \\
J \dot{\omega}+\omega^{\times} J \omega=L(\Delta \tau+\underbrace{\Delta \tau)+d}_{D} .
\end{gathered}
$$




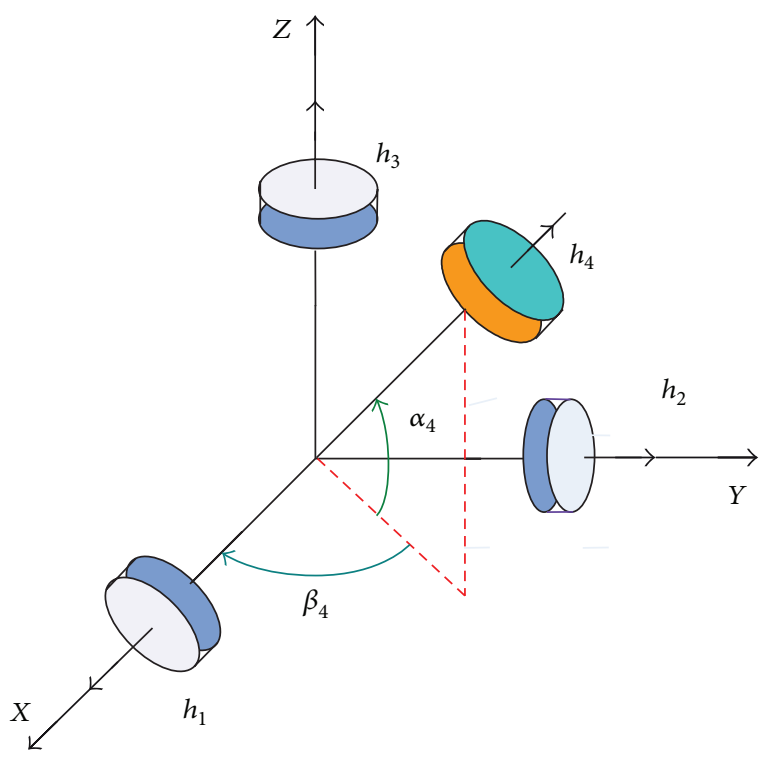

FIGURE 1: Configuration of four reaction wheels.

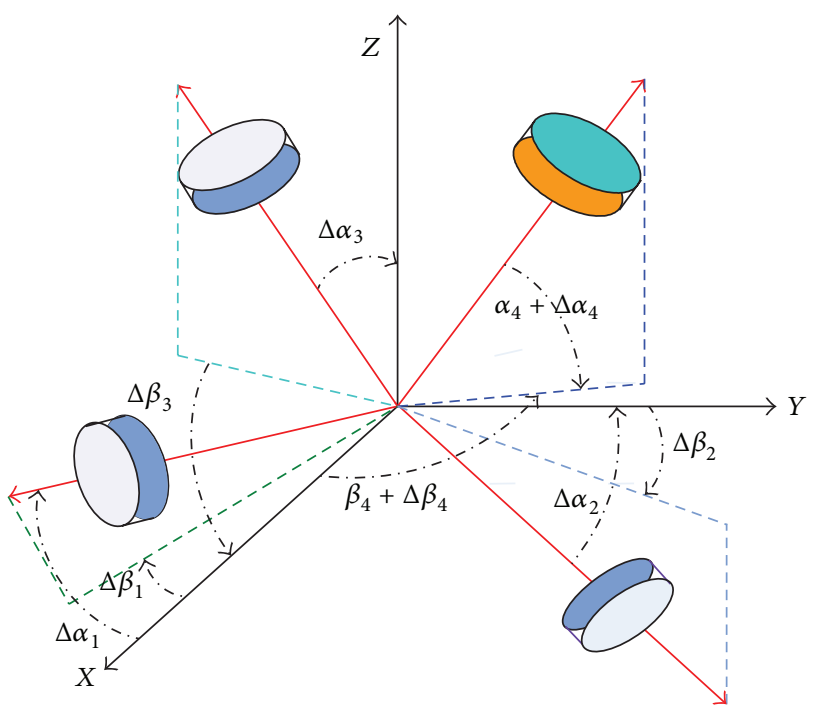

FIGURE 2: Four reaction wheels with misalignment.

2.4. Control Objective. To provide some insight into the control scheme, while also facilitating the subsequent stability analysis, we now define the filtered error variable [14]

$$
x=\left(\omega_{e}+\gamma e\right) \text {. }
$$

And the following (26) can be deduced via considering (8) and (10):

$$
J \dot{x}=\tau+P-\gamma e-\frac{1}{2} \dot{J} x,
$$

where $\gamma>0$ is a constant chosen by the user, and

$$
\begin{aligned}
P= & -\omega^{\times} J \omega+J\left[\left(\omega-R \omega_{d}\right)^{\times} R \omega_{d}-R \dot{\omega}_{d}\right] \\
& +d+\frac{\gamma}{2}\left(e^{\times}+e_{0} I_{3}\right) \omega_{e}+\gamma e+\frac{1}{2} \dot{J} x,
\end{aligned}
$$

where $P$ is the lumped term containing two part of system nonlinearities and external disturbances. The uncertain term is the key issue that needs to be solved for attitude tracking control design stems. We employ an indirect way to challenge the heavy computations, time-varying parameters which could be generated by the inappropriate means. The approach focuses on the core information $P$ itself and its bound that is used for the control design later. In this paper, we adopt this indirect method to deal with the effect of uncertainties and disturbances and to be an assistant for fault detection via robust adaptive control algorithms and the tracking performance index, as will be discussed later.

To this end, the control objective we want to achieve in this paper is to design a control law to realize stability attitude control about rigid spacecraft under faulty given by (1) and (27) such that the following goals are achieved in the presence of partial loss of actuator effectiveness fault and actuator failures.

(a) The closed-loop system is globally stable in which all the signals are bounded and continuous.

(b) The attitude orientation and angular velocity $\omega$ tracking errors converge to an arbitrary small set containing the origin in finite time $T_{0}$, that is, $\|e\| \leq \delta_{1}^{*}$ and $\left\|\omega_{e}\right\| \leq \delta_{2}^{*}$ for $t \geq T_{0}$.

(c) The tracking performance index (25) is bounded:

$$
I_{e}=\lim _{t \rightarrow \infty} \frac{1}{t} \int_{0}^{t}\left\|x\left(\omega_{e}, e\right)\right\|^{2} d t
$$

Remark 1. As we see clearly that the external disturbance $d$ is bounded and satisfies $\|d\| \leq r_{g}+r_{d}\|\omega\|^{2}$ with the unknown positive constant $r_{g}, r_{d}$, also note that $\left\|e^{\times}+e_{0} I_{3}\right\|=1$ and $\|R\|=1$.

Remark 2. Supposing that $\omega_{d}, \dot{\omega}_{d}, q_{d}, \dot{q}_{d}$ are all bounded for $t \geq 0$, then we have $\left\|\left(e^{x}+e_{0} I_{3}\right) \omega_{e}\right\|=\left\|\left(e^{x}+e_{0} I_{3}\right)(\omega-R) \omega_{d}\right\| \leq$ $\|\omega\|+r_{\omega}$ for an unknown constant $r_{\omega} \geq 0$. Also note that $\|\dot{J} x\| \leq r_{f}\left\|\left(\omega-R \omega_{d}\right)+\gamma e\right\| \leq r_{f}\|\omega\|+r_{0}$ for any constant $r_{0} \geq 0$.

Remark 3. Analysing above all, there always exist some unknown constants $a, a_{0}, a_{1}, a_{2}$ which are all the unknown constants and greater than zero, satisfying (30) and (31)

$$
\begin{gathered}
\|P\| \leq a_{0}+a_{1}\|\omega\|+a_{2}\|\omega\|^{2} \leq a \vartheta \\
\vartheta=1+\|\omega\|+\|\omega\|^{2} .
\end{gathered}
$$

It worth mentioning that both (30) and (31) are rigorously, regardless of external disturbances, uncertainties and timevarying moment inertia. Moreover, based on analysis above, 
this control algorithm of indirect method have so much merits such as simple structure, little online computations, as will be discussed in the next section. Moreover, the above control objective is to be guaranteed under the conditions of (a) possible actuator faults and (b) uncertain spacecraft inertia parameters and unknown bounded external disturbances. We present now the main results of this study.

\section{Control Law Design}

3.1. Adaptive Attitude Tracking Control. In this section, two steps are split for the controller using the principle of control allocation [28]. (1) Design a control law specifying which total control effort to be produced and (2) design a control allocator that maps the total control demand onto individual actuator settings, as shown in Figure 3. The control law design is under the cases (A) and (B): (A) actuator partial loss fault, and (B) actuator failures. And in this controller design, Cai's idea [14] is employed. In what follows, we will develop such an attitude control law for spacecraft as shown in (1) and (2) to achieve the control target given in Section 2.4.

(A) Attitude Tracking Control under Partial Loss Fault. When the spacecraft has endowed with three reaction flywheels and each of them partially loses its actuation effectiveness, the attitude dynamics are transformed from (1) to (32)

$$
J \dot{\omega}+\omega^{\times} J \omega=L \Gamma(\cdot) \tau+d
$$

where $\tau=\left[\begin{array}{lll}\tau_{1} & \tau_{2} & \tau_{3}\end{array}\right]^{T}, \Gamma(\cdot)=\operatorname{diag}\left(\rho_{1}, \rho_{2}, \rho_{3}\right) \in \mathbb{R}^{3 \times 3}$ with $0<\rho_{0} \leq \rho_{i} \leq 1$ being the actuator health indicator for the $i$ th actuator. The case $\rho_{i}=1$ implies that the $i$ th actuator is healthy, $\rho_{i}=0$ is the case in which the $i$ th actuator totally failed, and for $0<\rho_{0}<\rho_{i}<1$ corresponding to the case in which the $i$ th actuator partially loses its actuating power, but still works all the time. When the three reaction flywheels are in presence of partial power loss fault, the actuation effectiveness matrix $L^{\prime}$ remains positive definite, although it has become uncertainty and time varying. Before the redundancy flywheel is driven, in such case, we must ensure the vehicle attitude stability and reliability. The following result can guarantee this requirement.

Theorem 4. Consider a spacecraft endowed with three reaction flywheels involving partial loss fault in that the attitude dynamics are conducted by (32) with $0<\rho_{0} \leq \rho_{i} \leq 1$ ( $i=$ $1,2,3)$. If the control scheme is implemented as (33), then the control objectives as stated in (a) (b) (c) are achieved:

$$
\begin{gathered}
u=-\left[h_{0}+h\right] x, \quad h=\frac{\widehat{a} \vartheta}{\|x\|+\delta}, \\
\dot{\hat{a}}=-\sigma_{1} \widehat{a}+\sigma_{2} \frac{\|x\|^{2} \vartheta}{\|x\|+\delta}, \quad \delta=\frac{\eta}{1+\vartheta},
\end{gathered}
$$

where $h_{0}>0, \eta>0, \sigma_{1}>0, \sigma_{2}>0$ are selected by the designer.

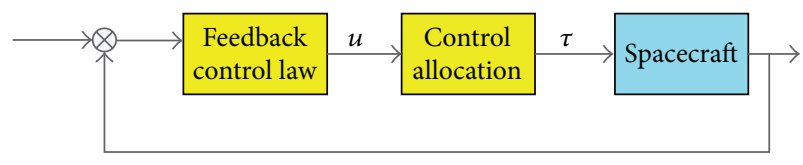

FIGURE 3: Block diagram for spacecraft attitude control with control allocation.

Remark 5. The control scheme stated as (33) is appropriate for the attitude tracking performance under the actuation effectiveness matrix $L^{\prime}$ with $0<\rho_{0} \leq \rho_{i} \leq 1$.

(B) Attitude Tracking Control under Actuator Error and Partial Loss Fault. At present, control efficiency matrix $L^{\prime}$ is supposed to be accurate and correct, Attitude tracking controller design under actuator failures is argued in this part. And the attitude dynamic is described by (27) which has been given before. For the same purpose as described in (A) it is interesting that this can be achieved with a slight modification to the control scheme presented earlier, as in Theorem 6.

Theorem 6. Consider the spacecraft with the attitude dynamics as in (27). If the following control scheme is employed

$$
\begin{gathered}
u=-\left[h_{0}+h\right] L^{T} x, \quad h=\frac{\widehat{a} \vartheta}{\|x\|+\delta}, \\
\dot{\hat{a}}=-\sigma_{1} \widehat{a}+\sigma_{2} \frac{\|x\|^{2} \vartheta}{\|x\|+\delta}, \quad \delta=\frac{\eta}{1+\vartheta}
\end{gathered}
$$

then, the stable attitude tracking is ensured even if all the flywheels suffer from actuator error and partial losing actuation $0<\rho_{0}<\rho_{i} \leq 1(i=1,2,3)$.

Theorems 4 and 6 are also cited from [14]; therefore, we omit the proof here.

Remark 7. It is clear that the control scheme only involves simple functions and variables as can be seen from (33) and (34). All can be lower computational cost and energy consumption.

Remark 8. It is worth mentioning that all the implicit assumption is the fact that the remaining actuation effectiveness is able to produce a sufficient actuating torque vector for the vehicle to perform the given maneuvers.

Remark 9. The reaction wheel distribution matrix $L$ is made full-row rank; the stability is ensured as long as $L \Gamma(\cdot) L^{T}$ is positive definite. In Cai' idea [14], $\lambda_{\min }\left(L \Gamma(\cdot) L^{T}\right)=0.5$ has proved that the control scheme is still able to ensure stable attitude tracking. Therefore, we set $\lambda_{\min }=0.5$ to be the threshold value.

Remark 10. It is worth mentioning that, although this work applied the same attitude tracking control approach as in [14] under the actuator fault and external disturbance, the main contribution of our study is the robust control allocation design in presence of actuator misalignment which will be discussed in Section 3.2. 
Although $L$ does not appear in the control scheme equation (34), to ensure the stability, reliability of the spacecraft attitude dynamics under actuator error with the fading actuation, we proposed a fault detection unit to identify or estimate the healthy condition of the flywheels. Once the instantaneous residual error $I_{e_{\text {_residual }}}$ exceeds the $\widehat{I}_{e_{\text {_residual }}}$ and meanwhile, the time holds $30 \mathrm{~s}$, the fault alarm is conveyed. Control allocation must be executed immediately.

\subsection{Robust Optimization Control Allocation under Control} Effectiveness Uncertainty. Based on the discussion above, the control allocation is generated under the uncertainty, such as the control effectiveness uncertainty caused by the partial power loss or actuator failure and even in presence of actuator misalignment. There is extensive literature on control allocation which discusses different algorithms, approaches, and applications [26, 28-33]. Recently, a robust optimization control allocation under uncertainty is generated. Therefore, we employ a novel robot control allocation algorithm combining with the optimization concept [34] to solve the problem. To facilitate discussion, we use $B$ to replace the $L E$ of (25) which takes into account the redundancy term this time. Then, the fault tolerant control allocation can be formulated as follow.

The relationship between the desired or commanded control input vector $\tau$ and actuator control input vector $u$ is formulated as

$$
\begin{aligned}
& u=B \tau_{c i} \\
& \text { s.t. } \quad \tau_{\min } \leq \tau_{c i} \leq \tau_{\max } \quad i=1,2,3,4,
\end{aligned}
$$

where $u$ represents the desired input, called "virtual control," from attitude controllers such as quaternion feedback control. The purpose of the control allocation is to find a feasible solution $\tau_{c i}$, to satisfy the control torque requirement in (50).

It is important to note that the problem of reconfiguring redundant actuators to tolerate any faulty actuators, such as external disturbance, actuator error, and partial power loss, is converted to the problem of finding the optimal input $u$ to solve tolerant fault control allocation. Robust optimization is focused on in this subsection and in order to give further explanation, the definition as follows is given firstly.

Definition 11. The optimization problem involves uncertain parameters can be described as

$$
\begin{array}{ll}
\min & f(z, \xi) \\
\text { s.t. } & g_{i}(z, \xi) \quad i=1,2,3,4,
\end{array}
$$

where $z$ is the decision variable, $f$ and $g_{i}$ present the objective function and constrain function, respectively, $\xi$ is the uncertain parameter, and $\Xi$ is the uncertain set. Then, (36) is the robust optimization problem under the $\Xi$ which is supposed to be a limited closed set.

The core ideology of the robust optimization problem is to convert the original problem with a certain extent approximation to a convex optimization problem with polynomial complexity. The key issue is to build the corresponding robust counterpart, and then the problem can be transformed into approximate robust problem via relevant optimization theory, and the optimal solution can be solved.

First, we construct the theoretical model of the robust control allocation problem according to the optimization problem. The dynamic control allocation, which recently was suggested by Härkegård [35] and can be seen as pursuing the minimum of the error and control power, is a solution of a constrained optimization problem stated as

$$
\begin{array}{ll}
\min _{u} & \left\{\left\|B \tau_{c i}-u\right\|_{1}-\left\|W_{\tau c i}-\tau_{p}\right\|_{1}\right\} \\
\text { s.t. } & \tau_{\min } \leq \tau_{c i} \leq \tau_{\max } \quad i=1,2,3,4 .
\end{array}
$$

It is readily transformed into the form of standard linear programming

$$
\begin{array}{ll}
\min _{z} & c^{T} z \\
\text { s.t. } & A z \geq b,
\end{array}
$$

where

$$
\begin{gathered}
c=\left[\begin{array}{ccc}
0 & 0 & 0 \\
w_{1} & w_{2} & w_{3} \\
1 & 1 & 1
\end{array}\right] \\
z=\left[\begin{array}{ccc}
\tau_{c i} & \Delta_{1} & \Delta_{2}
\end{array}\right]^{T} \\
A z=\left[\Delta_{1}, \Delta_{2}, \tau_{c i},-\tau_{c i},-B \tau_{c i}+\Delta_{2}, B \tau_{c i}\right. \\
\left.+\Delta_{2},-\tau_{c i}+\Delta_{1}, \tau_{c i}+\Delta_{2}\right]^{T} \\
b=\left[0,0,-\tau_{\max }, \tau_{\min },-u, u,-\tau_{P}, \tau_{P}\right]^{T}
\end{gathered}
$$

$w_{n},(n=1,2,3)$ is the diagonal element of the weighting matrix $W_{\tau c i}$, and $\Delta_{1}, \Delta_{2}$ are the deviation and the error relaxation factor, and $\tau_{P}$ is the ideal error which is usual set to zero. In the section, the arguing on the control allocation in presence of the control effectiveness matrix $B$ under uncertainty; therefore, the optimization problem of the control allocation can be stated as follows:

$$
\begin{array}{ll}
\min _{z} & c^{T} z \\
\text { s.t. } & a_{i} z \geq b_{i}, \quad \forall a_{i} \in \Xi_{i}, \quad i=1,2,3,4,
\end{array}
$$

where $a_{i}$ presents the $i$ th line of the matrix $B$ which involves the uncertain term.

In order to give further explanation, another two definitions as following are given, also. To facilitate comprehending the definitions, we firstly define the ellipsoid $V \in \mathbb{R}^{K}$

$$
V=\left\{\prod_{l}(v) \mid\|Q v\| \leq 1\right\},
$$

where $v \rightarrow \prod_{l}(v)$ is the affine from $\mathbb{R}^{L}$ to $\mathbb{R}^{K}$ and $Q$ is $M \times L$ matrix.

Definition 12. The uncertain set $\Xi \in \mathbb{R}^{4 \times 3}$ is the ellipsoidal uncertain set, if the following assumptions are all in existence. 
(a) For the given $Q_{l}$ and $\prod_{l}, \Xi$ is the intersection of the limited ellipsoids

$$
\Xi=\bigcap_{l=0}^{k} V\left(\prod_{l}, Q_{l}\right) .
$$

(b) $\Xi$ is bounded.

(c) The Slater conditions should be met, that is, there is at least a matrix $A \in \Xi$ belongs to the relative interior point of each ellipsoid

$$
\forall l \leq k \exists v_{l}: A=\prod_{l}\left(v_{l}\right), \quad\left\|Q_{l} v_{l}\right\|<1 ;
$$

then as for the control allocation issue (40), one can draw the conclusion as Theorem 13 which has been proved in [34].

Theorem 13. Equation (40) argues on the issue of the robust control allocation under ellipsoidal uncertainty; if the following two conditions (1) and (2) are all be met, then (40) is equivalent to an optimal problem on conic quadratic expressed by (45).

(1) Matrix $B$ row $a_{i}$ involves the uncertainty, but still in ellipsoid range

$$
\Xi_{i}=\left\{a_{i}: a_{i}=\bar{a}_{i}+\Theta_{i} v_{i},\left\|v_{i}\right\|_{2} \leq \varsigma\right\} \quad i=1, \ldots, M,
$$

where $\bar{a}_{i}$ is nominal value and $\Theta_{i}$ is symmetric positive semidefinite matrix.

(2) The assumptions of row $a_{i}$ are mutual independence;

$$
\begin{array}{ll}
\min _{z} & c^{T} z \\
\text { s.t. } & \bar{a}_{i}^{T} z-\varsigma\left\|\Theta_{i} z\right\|_{2} \geq b_{i}, \quad \forall i=1, \ldots, M .
\end{array}
$$

Using 2 order cone $K=\left\{\left(z_{1} ; \bar{z}\right) \in \mathbb{R}^{3}: z_{1} \geq\|\bar{z}\|, z_{1} \geq 0\right\}$ to define deflection relation expressed by $\geq K$ in $\mathbb{R}^{3}: z \geq K$, $y \Leftrightarrow z-y \geq K, 0 \Leftrightarrow z-y \in K$ where $z, y \in \mathbb{R}^{3}$, then Theorem 4 is generalized to the condition of the conic quadratic with uncertainty set.

Definition 14. Considering a system $S^{*}$ with limited vector inequality expressed via $A_{j}\left(\begin{array}{c}z \\ v\end{array}\right)-b_{j} \geq L_{j}^{m}$, when $Z$ is the mapping in the $\mathrm{x}$-plane of $S^{*}$ solution set, $Z \in \mathbb{R}^{3}$ can be expressed by conic quadratic inequality $C Q r$ and $S^{*}$ is the expression of $Z$.

Theorem 15 (see [36]). Considering the line control allocation with uncertainty as follows

$$
L P_{r}(\Xi)=\left\{\min _{z: A z \geq b} c^{T} z \mid(c, B, b) \in \Xi\right\}
$$

supposing the uncertain set $\Xi$ is expressed by

$$
\begin{gathered}
\xi=(c, A, B) \in \mathbb{R}^{3} \times \mathbb{R}^{4 \times 3} \times \mathbb{R}^{4} \\
\Xi=\left\{\xi \mid \exists v: A(\xi, v) \equiv P_{r} \xi+Q v+r \geq K 0\right\},
\end{gathered}
$$

where $A(\xi, v)$ is the affine mapping and $K$ is a 2 order cone direct product. Once the CQr of $\Xi$ is strictly feasible, then the robust control allocation with conic quadratic represented uncertainty set equals the issue of cone quadratic optimization as follows:

$$
\min \theta^{T} z^{*}\left\{\begin{array}{l}
a_{i}^{T} z^{*}-c_{i}-\xi^{T} r \geq 0 \\
A_{i}^{T} z^{*}-b_{i}-P_{r}^{T} \xi=0 \quad i=1,2,3,4,5, \\
Q^{T} \xi=0, \xi \geq K 0,
\end{array}\right.
$$

where $z^{*}, \xi_{1}, \xi_{2}, \xi_{3}, \xi_{4}, \xi_{5}$ are all the design variables, and $A_{i}$, $a_{i}, b_{i}, c_{i}$ are all from the affine functions $\alpha_{i}(\xi)=A_{i} \xi+a_{i}, \beta_{i}(\xi)=$ $b_{i}^{T} \xi+c_{i}$. And $P_{r}, Q, r$ come from the description of the following:

$$
(\Xi)=\left\{\xi \mid \exists v: P_{r} \xi+Q v+r \geq K 0\right\} .
$$

\section{Simulation Study}

The numerical application of the proposed control schemes to the attitude control of an orbiting spacecraft equipped with four reaction flywheels is presented using MATLAB/SIMULINK software. The complete set of physical parameters used in the numerical simulations is provided in Cai et al. [14], which is given by $J=J_{0}+\Delta J$, where $J_{0}=\left(\begin{array}{ccc}20 & 0 & 0.9 \\ 0 & 17 & 0 \\ 0.9 & 0 & 15\end{array}\right) \mathrm{kg} \cdot \mathrm{m}^{2}$ and an time-varying moment inertia matrix as stated in [38] is incorporated into the model

$$
\Delta J=1+e^{-0.1 t}+2 u^{*}(t-10)-4 u^{*}(t-20) \operatorname{diag}(3,2,1),
$$

where $u^{*}(\cdot)$ is defined as $u^{*}(t \geq 0)=1$ and $u^{*}(t<0)=0$. And the external torque disturbance is assumed to be

$$
d(t)=\left(\|\omega\|^{2}+0.005\right)\left[\begin{array}{lll}
\sin 0.8 t & \cos 0.5 t & \cos 0.3 t
\end{array}\right]^{T} \mathrm{~N} \cdot \mathrm{m}
$$

Moreover, the spacecraft is controlled by four reaction wheels with limited control torque $u_{\max }=5 \mathrm{~N} \cdot \mathrm{m}$, and the distribution matrix $L$ is calculated as

$$
L=\left[\begin{array}{cccc}
-1 & 0 & 0 & \frac{1}{\sqrt{3}} \\
0 & -1 & 0 & \frac{1}{\sqrt{3}} \\
0 & 0 & -1 & \frac{1}{\sqrt{3}}
\end{array}\right] .
$$

Here, a severe failure scenario is considered, where some flywheels partially lose their effectiveness and the effectiveness of the actuators are given by [14]

$$
\rho_{i}=0.7+0.15 \operatorname{rand}\left(t_{i}\right)+0.1 \sin \left(0.5 t+\frac{i \pi}{3}\right) \quad i=1, \ldots, 6
$$

where the definition of $\operatorname{rand}\left(t_{i}\right)$ is given in [14].

We simulate three different cases: (A) response of actuator partial lost fault case under external disturbances, (B) 


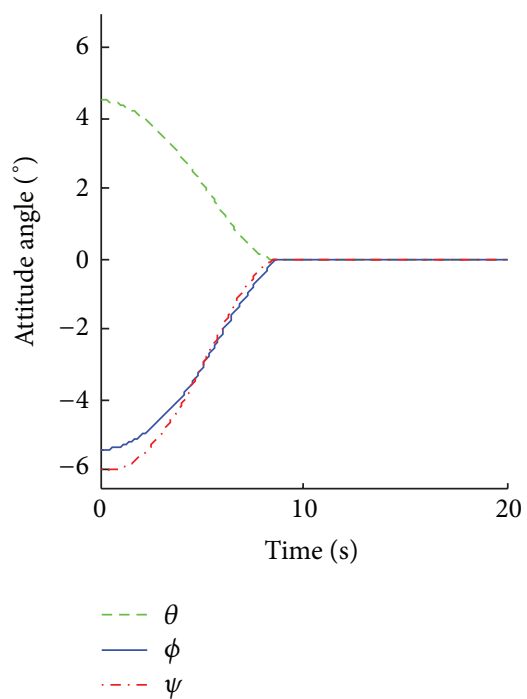

(a)

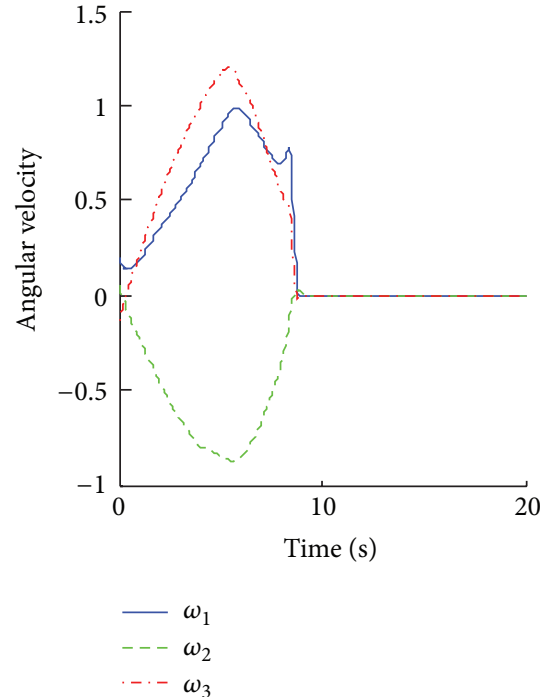

(b)

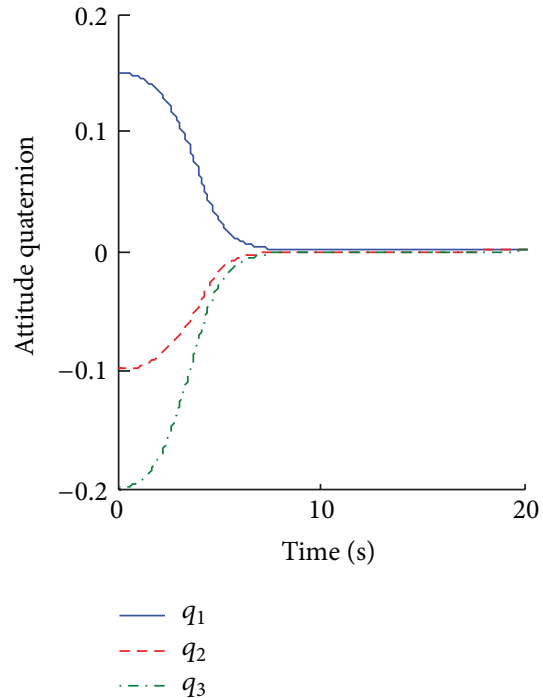

(c)

FIGURE 4: Time responses of the attitude angle, angular velocity, and attitude quaternion.

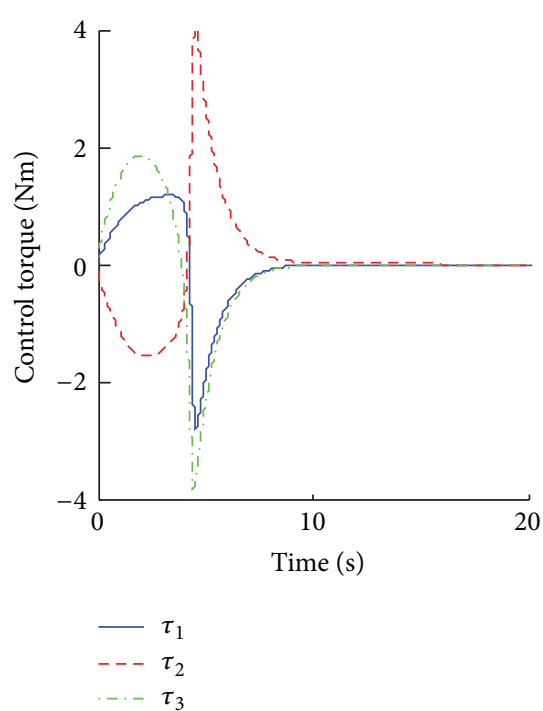

(a)

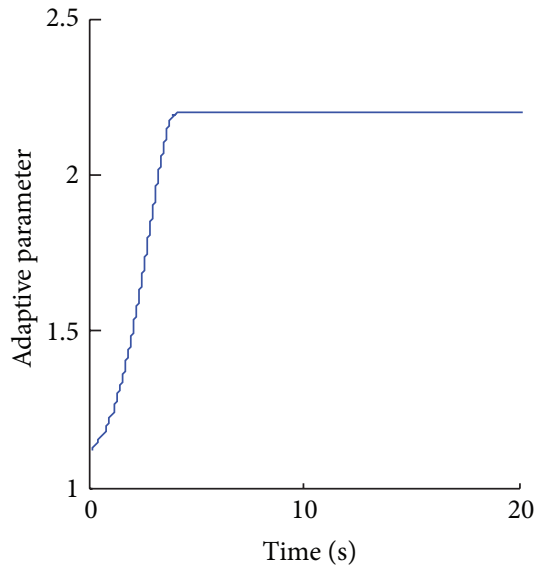

(b)

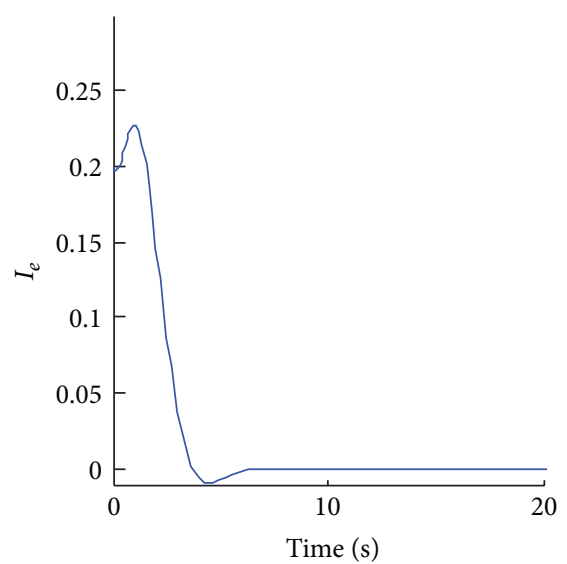

(c)

FIgURE 5: Three actuation torque $(\mathrm{N} \cdot \mathrm{m})$, adaptive parameter $\widehat{a}$, and bounded control performance index $I_{e}$.

response of actuator fault case under external disturbances, and (C) response of actuator fault case under external disturbances with uncertainty. Our earlier theoretical analysis declares that controller (30) can deal with the cases (A) and (B) if $\lambda_{\min }>0.5$. Otherwise, the robust control allocation is operated under Case (C). Among the three flywheels under the cases (A) and (B), the first flywheel only supplies 20 percent of its saturation value after 8 seconds, and the second and the third flywheels can supply $40 \%$ and $30 \%$ after 10 and 12 seconds, respectively. In this simulation, the control parameters are chosen quite arbitrarily and remain unchanged for all the simulation cases as $h_{0}=20, \sigma_{2}=100$, $\gamma=2, \sigma_{1}=0.01$, and $\eta=0.1$.

(A) Response of Actuator Partial Lost Fault Case under External Disturbances. In this case, (53) partial loss of actuator effectiveness fault under (51) external disturbances is considered. The faults are described by: at $t=8 \mathrm{~s}$, the first flywheel only supplies 20 percent of its saturation value, and the second and the third flywheels can supply $40 \%$ and $30 \%$ at 12 and 15 seconds, respectively. When the proposed controller in (33) and the fault detection scheme are all 


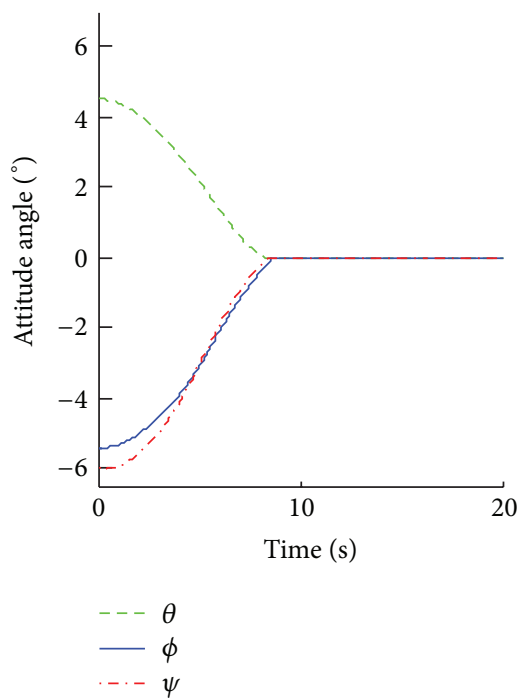

(a)

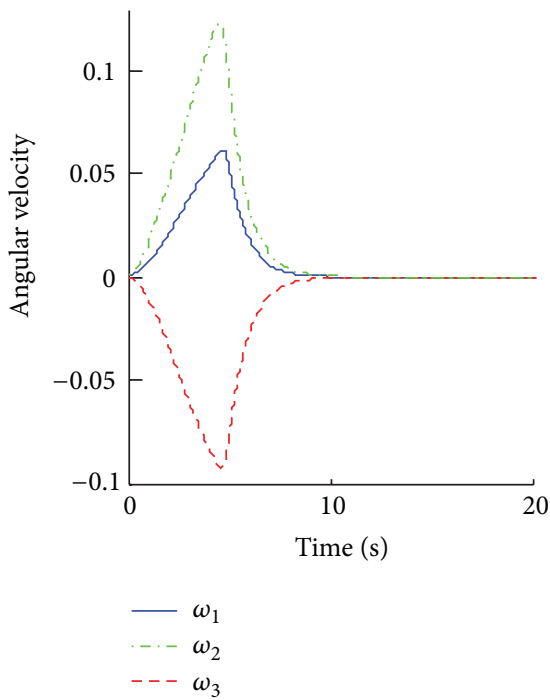

(b)

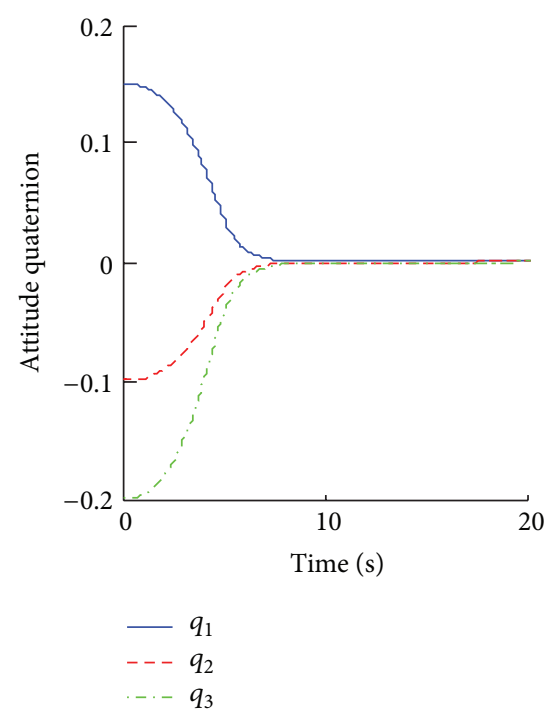

(c)

FIGURE 6: Time responses of the attitude angle, angular velocity, and attitude quaternion.

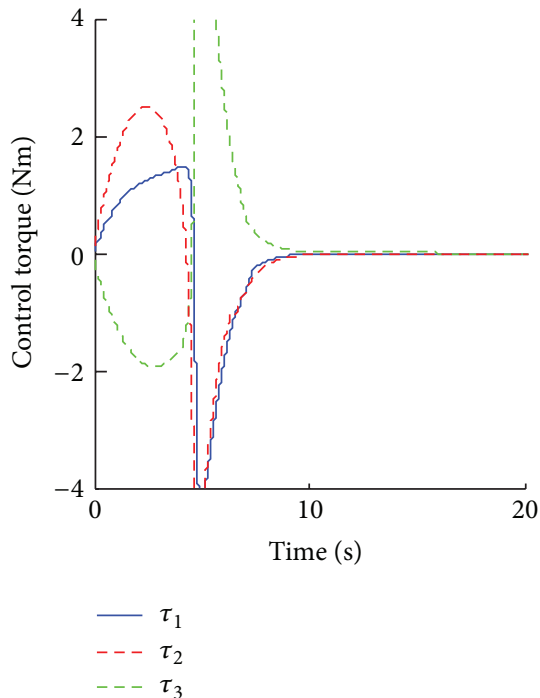

(a)

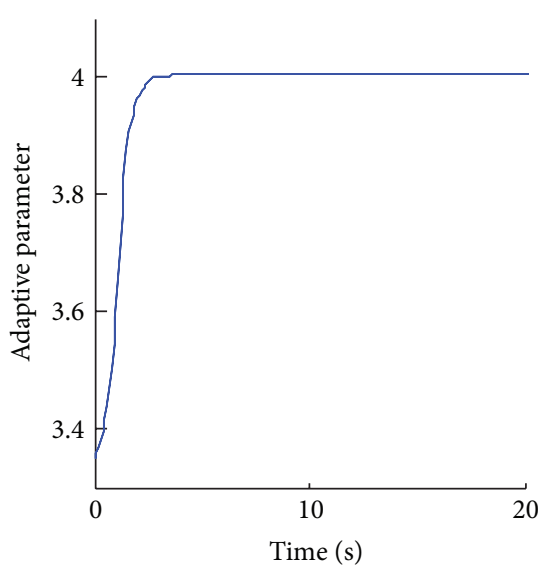

(b)

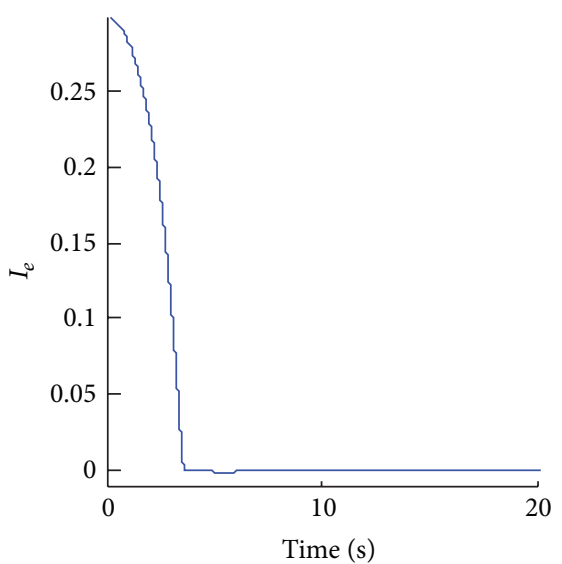

(c)

FiguRE 7: Three actuation torque $(\mathrm{N} \cdot \mathrm{m})$, adaptive parameter $\widehat{a}$, and bounded control performance index $I_{e}$.

implemented to the faulty attitude system, we can observe the tracking performance error via threshold curve $\widehat{I}_{e \text { _residual }}$. Once the fault is detected and the control allocation is generated.

The attitude stability of the spacecraft which enduring the actuator partial lost can be seen from the time responses of the attitude angle, angular velocity and attitude quaternion are presented in Figure 4, and the three actuation torque, adaptive parameter $\hat{a}$ and bounded control performance index $I_{e}$ are shown in Figure 5. It is seen that, from Figures 4 and 5, the control method has a fairly good control performance although in such severe flywheels faults with limited flywheel.

(B) Response of Actuator Fault Case under External Disturbances. In this case, the attitude controller design is under the actuator fault with the external disturbances with uncertainty. Equation (53) under (51) external disturbances is considered under the proposed control law equation (34). To prove the good attitude control performance, we give out the time responds of the attitude angle, angular velocity, attitude quaternion, three actuation torque, adaptive parameter $\widehat{a}$, and bounded control performance index $I_{e}$ which can be seen 


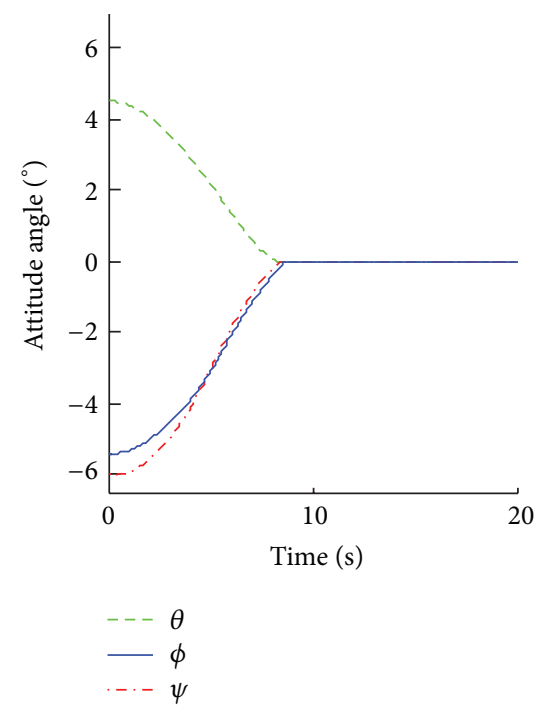

(a)

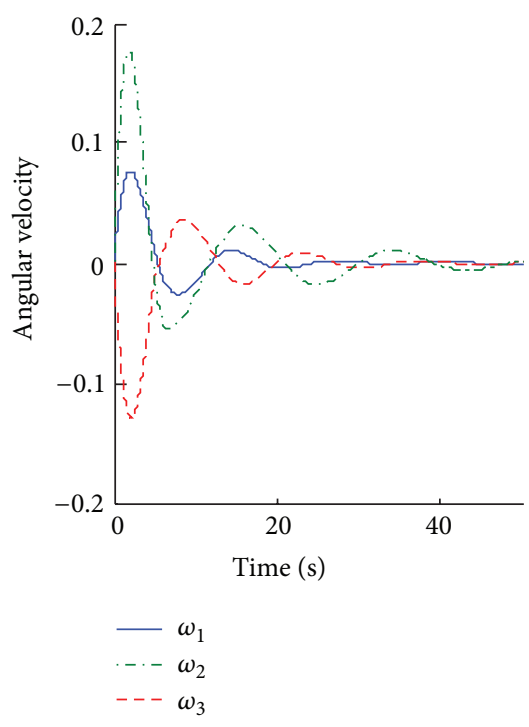

(b)

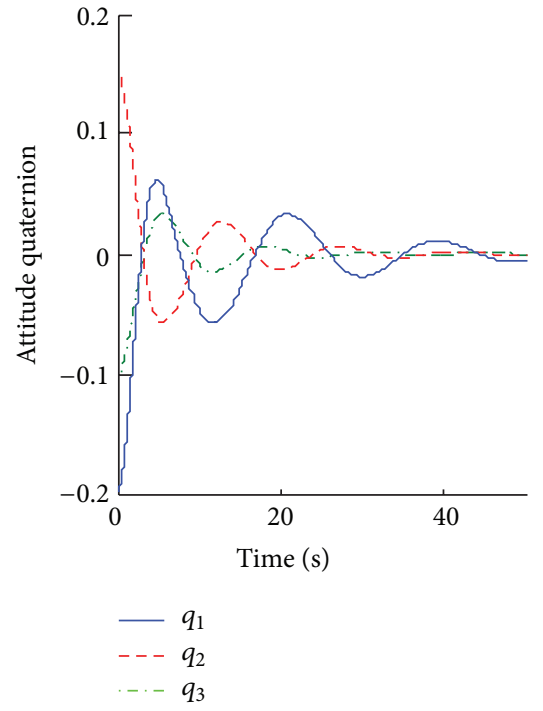

(c)

FIGURE 8: Time responses of the attitude angle, angular velocity, and attitude quaternion.

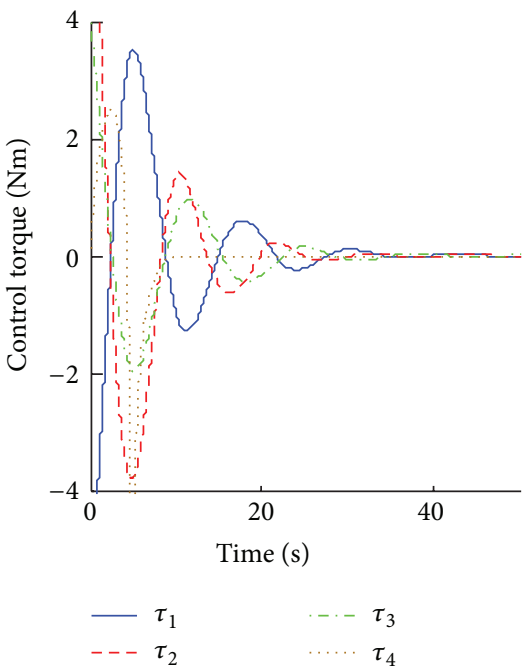

(a)

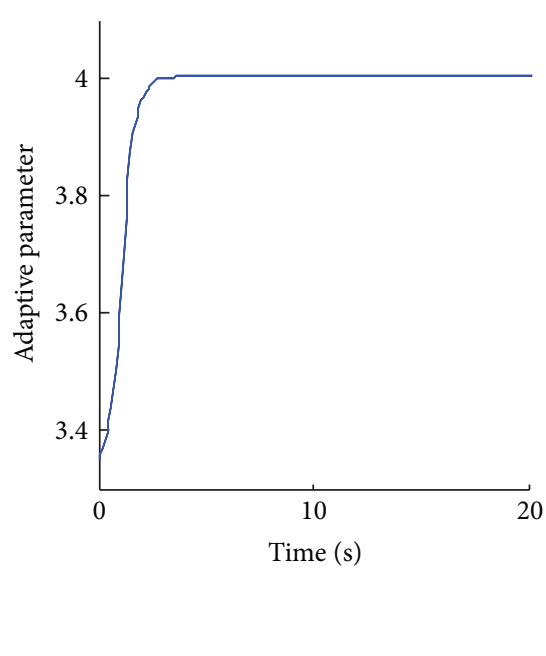

(b)

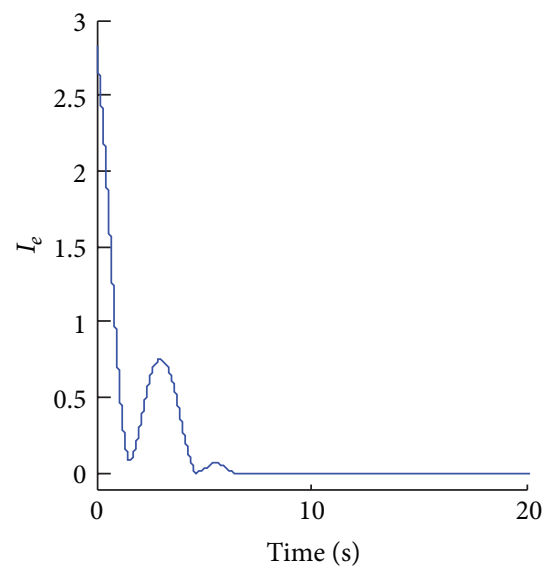

(c)

FIGURE 9: Three actuation torque $(\mathrm{N} \cdot \mathrm{m})$, adaptive parameter $\widehat{a}$, and bounded control performance index $I_{e}$.

from Figures 6 and 7. It is seen that, from Figures 6 and 7, the control method has a fairly good control performance although in such severe flywheels faults with limited flywheel.

(C) Response of Actuator Fault Case under External Disturbances with Uncertainty. This case not only involves partial losing power of the actuator under external disturbances which is discussed in (A) but also involves actuator failures discussed in (B). Moreover, actuator misalignment and magnitude error are considered here. The misalignment $\Delta \alpha_{i} \in$ $\left[-2^{\circ},+2^{\circ}\right](i=1,2,3), \Delta \beta_{i} \in\left[-180^{\circ},+180^{\circ}\right](i=1,2,3)$, $\Delta \alpha_{4}, \Delta \beta_{4} \in\left[-2^{\circ},+2^{\circ}\right]$ and the magnitude error could be expressed by (17). Similar conclusion can be deduced from Figures 8 and 9 . Once the fault detection system sent the information to generate control allocation the time responses of the attitude angle, angular velocity, and attitude quaternion for the unhealthy actuator case under the proposed controller (34), which has the same control parameters as controller (33), are used in the control scheme and are presented in Figure 8, and the time respond of control torque, adaptive parameter $\widehat{a}$, bounded control performance index $I_{e}$ are all presented in Figure 9. It is seen that, from Figures 8 and 9, the robust control allocation scheme has a fairly good fault tolerance performance. 


\section{Conclusions}

This study presented a robust fault tolerance control allocation strategy for rigid spacecraft attitude stabilization subject to actuator partial lost, actuator failures, and actuator misalignment. An adaptive attitude tracking strategy is employed which can realize fault tolerance control under the actuator partial loss and actuator failure within $\lambda_{\min }=$ 0.5. Meanwhile, the faults detection is always here during the procedure. Once the fault occurred which could not guaranteed the attitude stable for $30 \mathrm{~s}$, the robust control allocation strategy is generated automatically to realize tolerance. The robust control allocation compensates the control effectiveness uncertainty which caused the actuator misalignment. The unknown disturbances, uncertain inertia matrix, and even actuator error with limited actuators are all considered in the controller design process. All are achieved with inexpensive online computations. Numerical results are also presented in such a way that not only highlight the closed-loop performance benefits of the control law derived here but also illustrate its great robustness.

\section{Conflict of Interests}

The authors declare that there is no conflict of interests regarding the publication of this paper.

\section{Acknowledgments}

The present work was supported partially by National Natural Science Foundation of China (Project no. 61304149), Natural Science Foundation of Liaoning, China (Project no. 2013020044), and the Polish-Norwegian Research Programme operated by the National Centre for Research and 24 Development under the Norwegian Financial Mechanism 2009-2014 in the frame of Project Contract no. PolNor/200957/47/2013. The authors highly appreciate the above financial supports.

\section{References}

[1] Y. M. Zhang and J. Jiang, "Bibliographical review on reconfigurable fault-tolerant control systems," Annual Reviews in Control, vol. 32, no. 2, pp. 229-252, 2008.

[2] G. Pujol, "Reliable $H_{\infty}$ control of a class of uncertain interconnected systems: an LMI approach," International Journal of Systems Science, vol. 40, no. 6, pp. 649-657, 2009.

[3] S. Chen, G. Tao, and S. M. Joshi, "Adaptive actuator failure compensation designs for linear systems," International Journal of Control, Automation and Systems, vol. 2, no. 1, pp. 1-14, 2004.

[4] M. Benosman and K.-Y. Lum, "Application of absolute stability theory to robust control against loss of actuator effectiveness," IET Control Theory and Applications, vol. 3, no. 6, pp. 772-788, 2009.

[5] J. Cieslak, D. Henry, A. Zolghadri, and P. Goupil, "Development of an active fault-tolerant flight control strategy," Journal of Guidance, Control, and Dynamics, vol. 31, no. 1, pp. 135-147, 2008.
[6] G.-H. Yang and D. Ye, "Adaptive fault-tolerant $H_{\infty}$ control against sensor failures," IET Control Theory and Applications, vol. 2, no. 2, pp. 95-107, 2008.

[7] H. Alwi, C. Edwards, O. Stroosma, and J. A. Mulder, "Fault tolerant sliding mode control design with piloted simulator evaluation," Journal of Guidance, Control, and Dynamics, vol. 31, no. 5, pp. 1186-1201, 2008.

[8] M. L. Corradini, G. Orlando, and G. Parlangeli, "A fault tolerant sliding mode controller for accommodating actuator failures," in Proceedings of the 44th IEEE Conference on Decision and Control, and the European Control Conference (CDC-ECC '05), pp. 3091-3096, New York, NY, USA, December 2005.

[9] G. J. J. Ducard, Fault-Tolerant Flight Control and Guidance Systems: Practical Methods for Small Unmanned Aerial Vehicles, Springer, London, UK, 2009.

[10] X. D. Zhang, T. Parisini, and M. M. Polycarpou, "Adaptive fault-tolerant control of nonlinear uncertain systems: an information-based diagnostic approach," IEEE Transactions on Automatic Control, vol. 49, no. 8, pp. 1259-1274, 2004.

[11] Y. Kobayashi, M. Ikeda, and Y. Fujisaki, "Stability of large space structures preserved under failures of local controllers," IEEE Transactions on Automatic Control, vol. 52, no. 2, pp. 318-322, 2007.

[12] S. Varma and K. D. Kumar, "Fault tolerant satellite attitude control using solar radiation pressure based on nonlinear adaptive sliding mode," Acta Astronautica, vol. 66, no. 3-4, pp. 486-500, 2010.

[13] G. A. Boyarko, M. Romano, and O. A. Yakimenko, "Timeoptimal reorientation of a spacecraft using an inverse dynamics optimization method," Journal of Guidance, Control, and Dynamics, vol. 34, no. 4, pp. 1197-1208, 2011.

[14] W. C. Cai, X. H. Liao, and Y. D. Song, "Indirect robust adaptive fault-tolerant control for attitude tracking of spacecraft," Journal of Guidance, Control, and Dynamics, vol. 31, no. 5, pp. 1456-1463, 2008.

[15] S. Yin, S. X. Ding, A. H. A. Sari, and H. Hao, "Data-driven monitoring for stochastic systems and its application on batch process," International Journal of Systems Science, vol. 44, no. 7, pp. 1366-1376, 2013.

[16] S. Yin, S. X. Ding, A. Haghani, H. Hao, and P. Zhang, "A comparison study of basic data-driven fault diagnosis and process monitoring methods on the benchmark Tennessee Eastman process," Journal of Process Control, vol. 22, no. 9, pp. 1567-1581, 2012.

[17] S. Yin, H. Luo, and S. Ding, "Real-time implementation of faulttolerant control systems with performance optimization," IEEE Transactions on Industrial Electronics, vol. 61, no. 5, pp. 24022411, 2013.

[18] S. Yin, X. Yang, and H. R. Karimi, "Data-driven adaptive observer for fault diagnosis," Mathematical Problems in Engineering, vol. 2012, Article ID 832836, 21 pages, 2012.

[19] Y. Jiang, Q. L. Hu, and G. F. Ma, "Adaptive backstepping faulttolerant control for flexible spacecraft with unknown bounded disturbances and actuator failures," ISA Transactions, vol. 49, no. 1, pp. 57-69, 2010.

[20] T. Jiang and K. Khorasani, "A fault detection, isolation and reconstruction strategy for a satellite's attitude control subsystem with redundant reaction wheels," in Proceedings of the IEEE International Conference on Systems, Man, and Cybernetics (SMC '07), pp. 3146-3152, Montreal, Canada, October 2007.

[21] J. J. Liang, C. Y. Dong, and Q. Wang, "A fault-tolerant attitude control system for a satellite based on fuzzy global sliding 
mode control algorithm," in 7th International Symposium on Instrumentation and Control Technology, Proceedings of SPIE, no. 12827, Beijing, China, 2008.

[22] X. Xie, S. Yin, H. Gao, and O. Kaynak, "Asymptotic stability and stabilisation of uncertain delta operator systems with timevarying delays," IET Control Theory \& Applications, vol. 7, no. 8, pp. 1071-1078, 2013.

[23] Q. Hou, Y. H. Cheng, N. Y. Lu, and B. Jiang, "Study on FDD and FTC of satellite attitude control system based on the effectiveness factor," in Proceedings of the 2nd International Symposium on Systems and Control in Aerospace and Astronautics (ISSCAA '08), pp. 1096-1101, Shenzhen, China, December 2008.

[24] H. Alwi and C. Edwards, "Fault tolerant control using sliding modes with on-line control allocation," in Fault Tolerant Flight Control, pp. 247-272, Springer, 2010.

[25] J. Buffington, P. Chandler, and M. Pachter, "On-line system identification for aircraft with distributed control effectors," International Journal of Robust and Nonlinear Control, vol. 9, pp. 1033-1049, 1999.

[26] Y. Choi, H. Bang, and H. Lee, "Dynamic control allocation for shaping spacecraft attitude control command," in Proceedings of the AIAA Guidance, Navigation, and Control Conference, vol. 6040, pp. 39-53, August 2006.

[27] R. Kristiansen, A. Loría, A. Chaillet, and P. J. Nicklasson, "Spacecraft relative rotation tracking without angular velocity measurements," Automatica, vol. 45, no. 3, pp. 750-756, 2009.

[28] O. Härkegård, Backstepping and Control Allocation With Applications to Flight Control, Linköpings University, 2003.

[29] M. Bodson and S. A. Frost, "Control allocation with load balancing," in AIAA Guidance, Navigation, and Control Conference \& Exhibit, August 2009.

[30] J. J. Burken, P. Lu, Z. Wu, and C. Bahm, “Two reconfigurable flight-control design methods: robust servomechanism and control allocation," Journal of Guidance, Control, and Dynamics, vol. 24, no. 3, pp. 482-493, 2001.

[31] T. I. Fossen and T. A. Johansen, "A survey of control allocation methods for ships and underwater vehicles," in Proceedings of the 14th Mediterranean Conference on Control and Automation (MED '06), June 2006.

[32] O. Härkegård, "Dynamic control allocation using constrained quadratic programming," Journal of Guidance, Control, and Dynamics, vol. 27, no. 6, pp. 1028-1034, 2004.

[33] Y. Luo, A. Serrani, S. Yurkovich, D. B. Doman, and M. W. Oppenheimer, "Model predictive dynamic control allocation with actuator dynamics," in Proceedings of the American Control Conference (AAC '04), pp. 1695-1700, July 2004.

[34] J.-J. Ma, W.-Q. Li, Z.-Q. Zheng, and D.-W. Hu, "Control of allocation under uncertainty based on robust optimization," Control Theory and Applications, vol. 27, no. 6, pp. 731-744, 2010.

[35] O. Härkegård, "Efficient active set algorithms for solving constrained least squares problems in aircraft control allocation," in Proceedings of the 41st IEEE Conference on Decision and Control, pp. 1295-1300, December 2002.

[36] Z.-S. Duan and C.-Z. Han, "A strong tracking adaptive state estimator and simulation," Acta Simulata Systematica Sinica, vol. 5, p. 41, 2004.

[37] K. Subbarao and V. R. Akella, "Differentiator-free nonlinear proportional-integral controllers for rigid-body attitude stabilization," Journal of Guidance, Control, and Dynamics, vol. 27, no. 6, pp. 1092-1096, 2004.
[38] Y. D. Song and W. C. Cai, "Quaternion observer-based modelindependent attitude tracking control of spacecraft," Journal of Guidance, Control, and Dynamics, vol. 32, no. 5, pp. 1476-1482, 2009. 


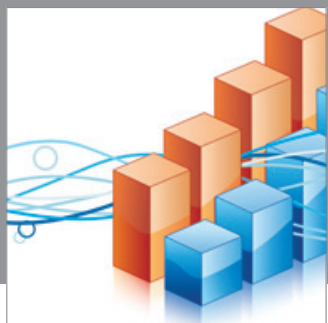

Advances in

Operations Research

mansans

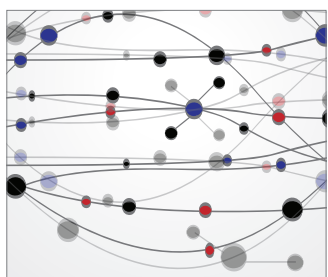

The Scientific World Journal
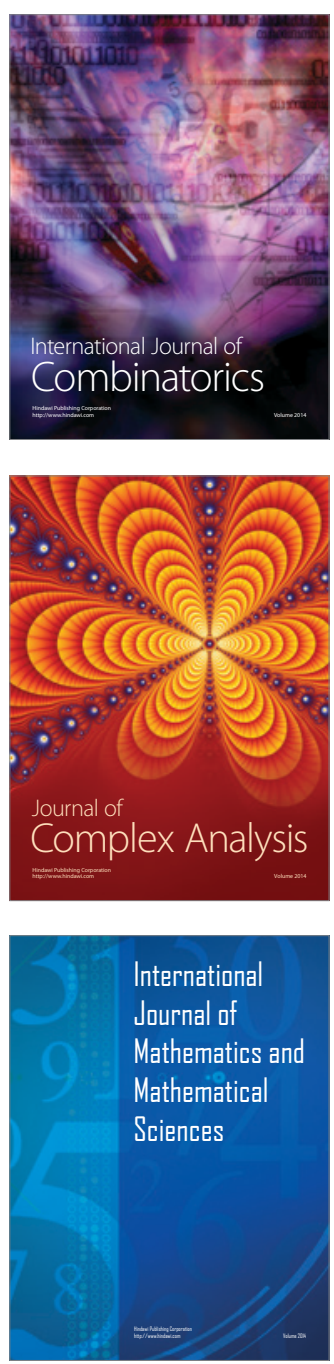
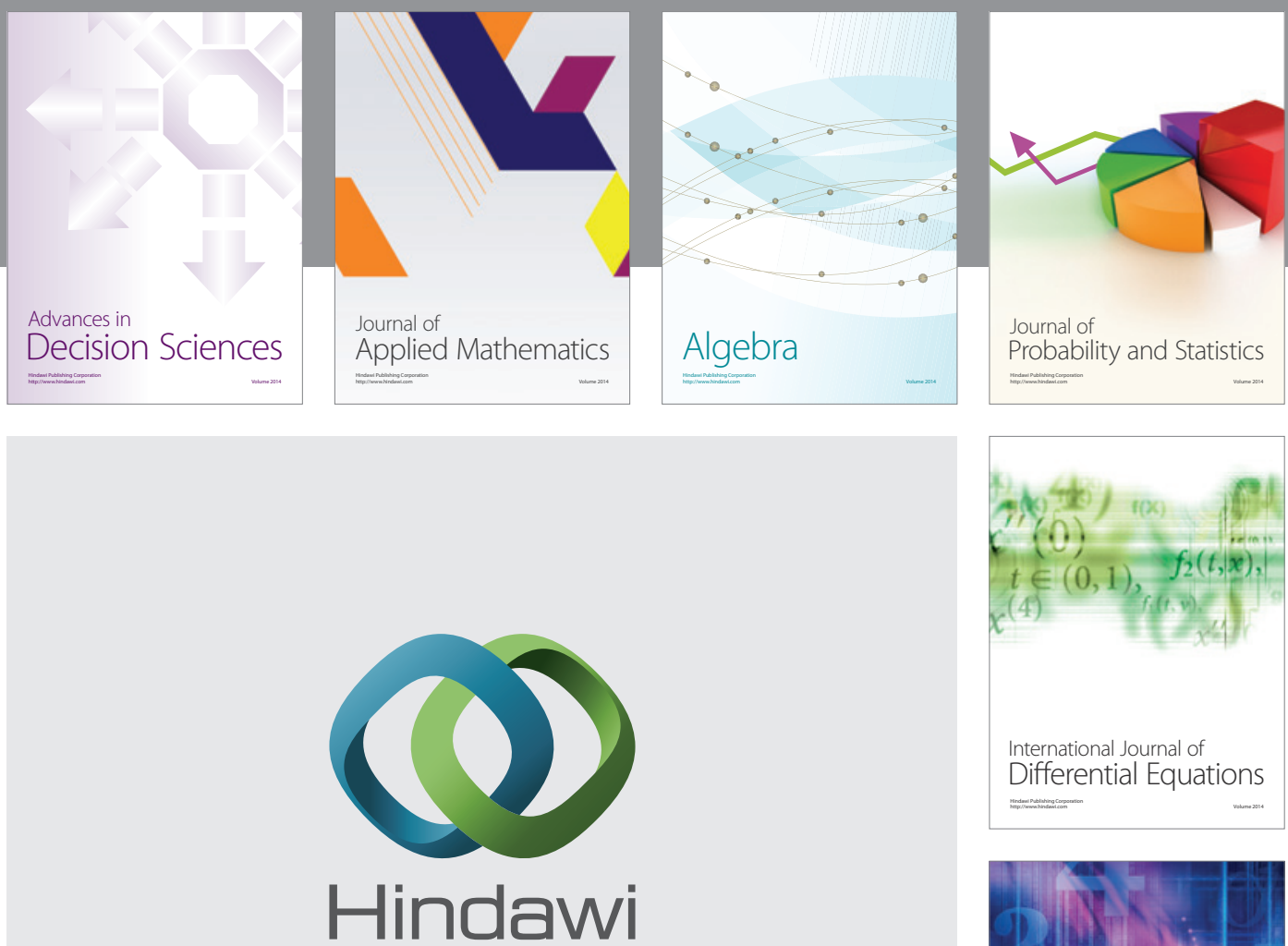

Submit your manuscripts at http://www.hindawi.com
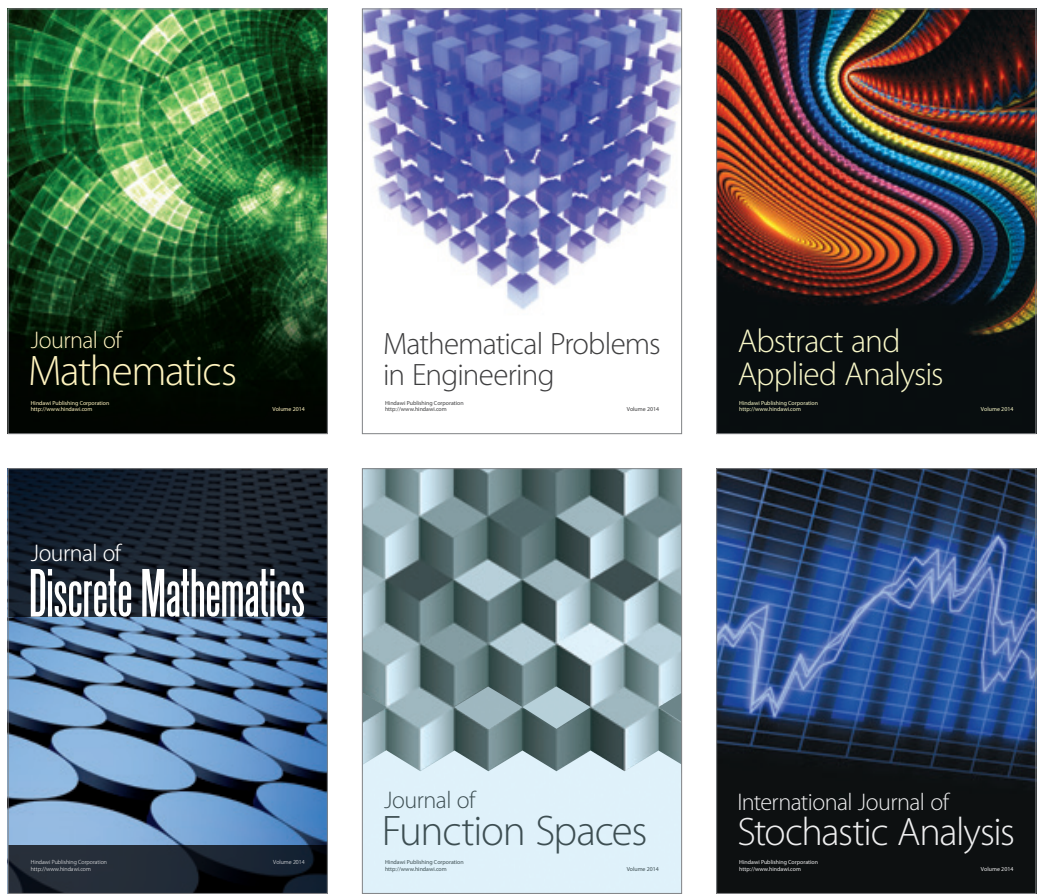

Journal of

Function Spaces

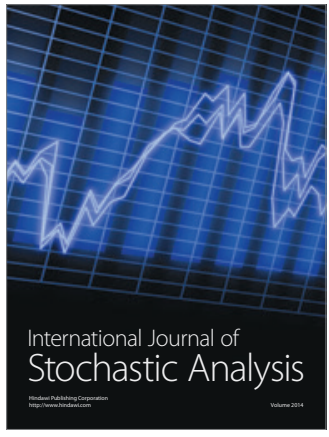

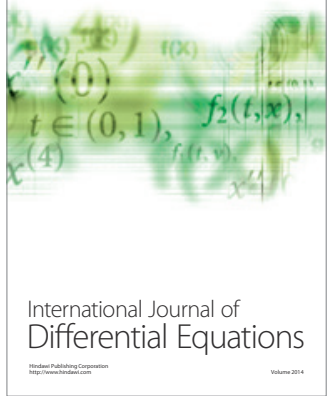
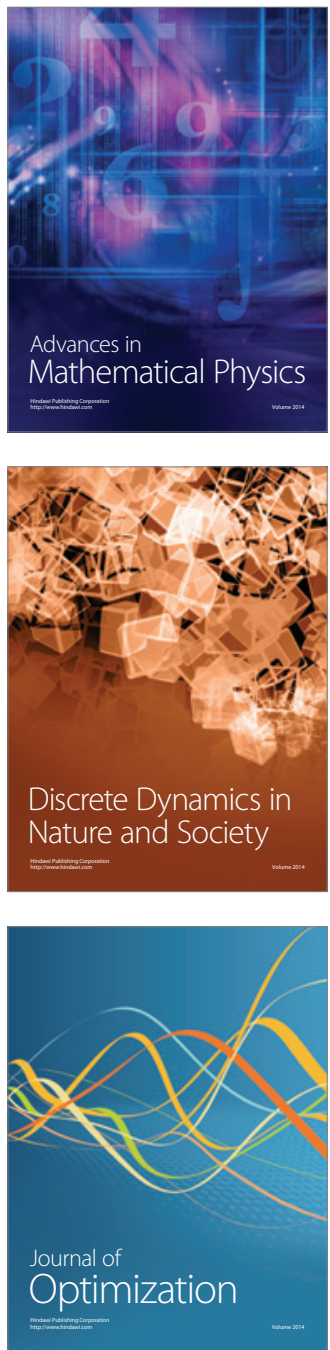